\title{
Non-Delaminating Pulsatile Release Composites
}

\author{
By Swapnil Gandhi and Eric Nuxoll* \\ Department of Chemical and Biochemical Engineering \\ 4133 Seamans Center for the Engineering Arts \& Sciences \\ University of Iowa \\ Iowa City, IA 52242, U.S.A. \\ *corresponding author \\ email: eric-nuxoll@uiowa.edu \\ office: 1-319-353-2377 \\ fax: $1-319-335-1415$
}

Keywords: Controlled Release, Barrier Membrane, Stimuli-sensitive Hydrogel, Computational Modeling

\begin{abstract}
By sequestering individual doses of chemical in stimuli-sensitive depot membranes and stacking them between stimulant barriers, automated pulsatile chemical release can be obtained from a simple polymer laminate. By using non-degrading hydrogel depots, this approach has been demonstrated releasing multiple chemicals in up to ten pulses, with each depot membrane delaminating and releasing its payload at a preprogrammed time. This paper reports the first experimental demonstration of a non-delaminating, non-degrading pulsatile release composite, for applications where delamination may be unsuitable. Non-delaminating systems not only require significantly larger development resources, they have an inherent periodicity limit which increases with the number of pulses desired. This limit is not analytically tractable, prompting the development of a computational model to predict the complete release profile. This model is validated against experimental delaminating systems, then adapted to nondelaminating systems to correlate the system's pulsatility with the physical parameters of the system, including diffusion coefficients, stimulant concentration, solute loading, scavenger loading and membrane thicknesses. These correlations facilitate rapid feasibility assessment of proposed non-delaminating pulsatile release applications prior to development of physical systems.
\end{abstract}




\section{Introduction.}

Pulsatile solute delivery is important to many fields, most notably medical applications such as hormone therapies(Bhalla and Siegel, 2014; Brabant et al., 1992; Hayes and Crowley, 1998), vaccines(Centers for Disease and Prevention, 2009; Walters et al., 2015), cancer therapies(Innominato et al., 2014; Lévi et al., 1997), and allergy treatments(Li et al., 2003), as well as for drugs where tolerance development is a concern, such as opiates(McKim, 2007) or nitroglycerin(Abrams, 1988). The ability to sequentially deliver multiple chemicals in a single administration, as well as minimize overall chemical consumption, also motivate the development of pulsatile delivery systems. While manual application of individual pulses is straightforward, automated pulsatile release typically involves pumps, power supplies, and circuitry at considerable expense and with many possible failure modes. Most materials-based approaches, such as time-release capsule sets(Gazzaniga et al., 2008; Tang et al., 2005; Thitinan and McConville, 2012), are constrained to two or three distinct pulses. Slight variations from capsule to capsule within a set lead to broad pulses, and as each capsule set operates independently to deliver a single pulse, slight variations in timing control quickly lead to overlapping pulses.

By coupling the release of one pulse to the release of the next, this problem can be overcome. Rather than sequester individual doses of chemical in independent sets of capsules, they can each be sequestered in a stimuli-sensitive depot membrane. By stacking these membranes in a single laminate, interlaced with barrier membranes to provide the delay time between each pulse, multiple distinct pulses can be released from a single polymer chip. This approach, termed Barrier-Mediated Pulsatile Release (BMPR), was initially proposed using water as a stimulant blocked by hydrolytically degradable polymer barriers(Fujioka et al., 1987; 
Jeon and Puleo, 2008; Jiang and Zhu, 2000; Liu et al., 2007). Once the water penetrated a barrier, it would dissolve and release the underlying depot, then begin hydrolyzing the next barrier. The stability and scaling constraints of this system initially limited its use; however, it has recently been generalized to employ hydrogel depots which can be triggered by a variety of stimulants. Devices releasing up to ten pulses have been demonstrated, with different chemicals in each pulse(Gandhi et al., 2015). By constructing barrier films from highly permeable hydrogel loaded with a sacrificial scavenger of the stimulant, the delay time for each pulse can be scaled precisely across orders of magnitude by varying the scavenger loading and the barrier membrane thickness.

To date, BMPR research has focused exclusively on delaminating systems where each barrier and depot are eliminated upon releasing their payload, either by dissolution/degradation or by physical separation from the stack. This allows rapid chemical release from the triggered depot and exposes the next barrier to the same constant stimulant source, making analytical prediction of the delay time straightforward. It also, however, makes the device difficult to recover and remove if necessary. For applications where the stack must remain intact, the performance of the device will change significantly, as depicted in Figure 1. Stimulant transport across the second barrier will be slowed by transport resistance from the spent membranes of the first pulse. This added transport resistance will also slow the release of solute from the second depot and broaden its release pulse. Subsequent pulses will undergo even more spreading, until the pulses overlap and the release is no longer truly pulsatile. Moreover, these incremental increases in mass transfer resistance also make analytical prediction of the release profile intractable. 
This paper demonstrates this effect experimentally using two-pulse non-delaminating BMPR systems, then reports the development of a computational model for predicting the release profile of non-delaminating systems. This model is then used to develop analytical correlations to quickly predict the pulsatile behavior of non-delaminating BMPR systems based on their transport and design parameters.

\section{Materials and Methods.}

\subsection{Materials.}

Methyl methacrylate (MMA), 2-dimethylamino ethyl methacrylate (DMA), and divinyl benzene (DVB) were obtained from Aldrich (Milwaukee, WI). Polymerization inhibitors in these monomers were removed by adsorption on alumina (Fisher Scientific, Pittsburgh, PA); the monomers were then refrigerated until used. 2, 2'-Azobisisobutyronitrile (AIBN), zinc oxide ( $\mathrm{ZnO})$, citric acid, sodium hydroxide, and potassium chloride were obtained from Sigma-Aldrich and used as received. Monobasic potassium phosphate and sodium chloride were used as received from Research Products International (Mt. Prospects, IL). Poly (vinyl alcohol) (PVA, 99\% hydrolyzed, MW 133,000) was obtained from Polysciences (Warrington, PA) and used as received. Methylene blue (MB, Sigma-Aldrich) was ground with a mortar and pestle then screened at 100 mesh before use.

\subsection{Demonstration of physical non-delaminating BMPR system.}

For experimental proof-of-principle, a non-delaminating BMPR device was developed by adaptation of a previously reported delaminating BMPR system(Gandhi et al., 2015). The depot membranes were comprised of $\mathrm{pH}$-sensitive poly(methyl methacrylate-co-dimethylamino ethyl methacrylate) (PMMA/DMA). At pH 7, this polymer remains glassy and hydrophobic, effectively immobilizing any chemical loaded into it. At $\mathrm{pH} 3$, however, the polymer swells 
rapidly with water, allowing the dissolution and diffusional release of the chemical(Firestone and Siegel, 1991; Siegel and Firestone, 1988). To protect the depot from acid stimulant for a fixed period of time, barrier membranes comprised of poly(vinyl alcohol) (PVA) loaded with zinc oxide nanoparticles were placed above each depot membrane(Nuxoll and Cussler, 2005; Nuxoll et al., 2005). This stack of alternating barrier and depot membranes was sealed around the edges and bottom and immersed in acid. After consuming the $\mathrm{ZnO}$ in the first barrier membrane, the acid would reach the underlying depot membrane and protonate the amine groups in the copolymer, prompting the depot membrane to swell with water. Once swollen, the depot membrane would release its payload and facilitate acid transport into the next barrier membrane. Barrier and depot membranes for the non-delaminating BMPR system were fabricated as previously reported(Gandhi et al., 2015). Barrier membranes were formed from $10 \%(\mathrm{w} / \mathrm{w})$ PVA solution with $\mathrm{ZnO}$ nanoparticles dispersed by stirring and sonication. After cooling, the suspension was cast on a poly(tetrafluoroethylene) (PTFE) sheet and calendared to the desired thickness. After at least 24 hours of drying under ambient conditions, the membranes were removed from the substrate, clamped between glass plates, and cured at $150{ }^{\circ} \mathrm{C}$ under vacuum for two hours to crosslink. Films were stored under ambient conditions then immersed in water to hydrate for at least 24 hours before use. Complete details of the depot membrane fabrication are given in Section S1 of the Supplementary Information. Briefly, depot membranes were polymerized from a 72:28 molar ratio of methyl methacrylate:dimethylamino ethyl methacrylate using 2,2 '-azobisisobutyronitrile as an initiator. This system was polymerized with stirring at 80 ${ }^{\circ} \mathrm{C}$ on a hot plate until the solution reached a viscosity of approximately $65 \mathrm{cP}$ (approximately 45 minutes), then quickly chilled to below room temperature. Methylene blue powder (the chemical released in these studies) was dispersed into this oligomer solution and the suspension was then 
cast between silanized glass plates separated by a PTFE spacer which determined the membrane thickness. The solution was mounted vertically in a vacuum oven and cured for 18 hours under nitrogen at $60{ }^{\circ} \mathrm{C}$. Unlike the delaminating BMPR system, however, where a large swelling pressure is desirable to reliably delaminate the depot from the rest of the stack, in a nondelaminating system, swelling must be optimized to minimize delamination stresses while still allowing rapid dissolution and diffusion of the embedded chemical. For this system, the divinyl benzene cross-linker content was increased from $0.1 \mathrm{wt} \%$ to $1.0 \mathrm{wt} \%$. Trials demonstrating that this change in crosslinker content reduces the equilibrium swelling ratio by half without inhibiting chemical release are described in Section S2 of the Supplementary Information.

Figure 2 shows a schematic of the non-delaminating BMPR system, alongside a physical device. Two 1-cm-wide depot membranes were each covered by a 1.2-cm-wide barrier membrane and stacked vertically atop a PTFE plug, then covered with a $150 \mu \mathrm{m}$ metal mesh. Melted beeswax was poured around the perimeter of the stack and cooled, sealing the edges and locking the metal mesh in place. The bottom of the device was coated with flexible rubber cement, allowing the stack to expand downward as the depot membranes swelled without delaminating from the system. Devices were immersed in $0.01 \mathrm{M} \mathrm{pH} 7$ phosphate buffer for a day before immersion in $0.01 \mathrm{M} \mathrm{pH} 3$ citrate buffer. Methylene blue release was monitored using a fiber-optic UV/Vis probe (Cary 50, Varian Inc., Mulgrave, Austrailia).

\subsection{Construction of BMPR computational model}

Construction of the computational model for non-delaminating BMPR systems was performed in stages. First, a computational model of a delaminating BMPR system was constructed. Several performance characteristics of this system such as barrier lag time can be calculated analytically, allowing simple precise validation of the computational methods. 
Moreover, these systems are much more experimentally accessible, allowing experimental validation of the assumptions used in model construction. Once the delaminating computational model was analytically and experimentally validated, it was adapted for non-delaminating systems. The model was constructed in one dimension using finite difference calculations, with each membrane divided into $n$ equal layers along its thickness, each represented by a node located at the upstream (exterior-facing) edge of the layer, as indicated in Figure 3. One additional node was located at the downstream edge of the device.

\subsubsection{Model assumptions.}

Several material assumptions were required to make computational modeling of the experimental BMPR system tractable. First, the swelling of the depot hydrogel and the release of the embedded solute are both assumed to be transport-limited. Previous studies have demonstrated that the polymer relaxation time is negligible compared to the acid diffusion time, and that the chemical dissolution time is negligible compared to its diffusion time(Firestone and Siegel, 1988, 1991; Gandhi et al., 2015). Second, the hydrogel exists in one of only two states: shrunken or swollen. In its shrunken state it is glassy and all diffusion coefficients are effectively zero, while in its swollen state its physical parameters match those of the PMMA/DMA membrane in $\mathrm{pH} 3$ buffer. Importantly, the stimulant is still capable of entering the shrunken depot to swell it, as non-dissociated weak acids can partition into hydrophobic polymers. Third, the stimuli-sensitive moiety in the depot membrane stoichiometrically consumes the entering stimulant instantaneously and irreversibly. This eliminates the need to track the acid concentration within a shrunken depot node. By constructing a delaminating computational model using these assumptions and comparing it against experimental results, the validity of the assumptions was tested. 


\subsubsection{Delaminating model construction.}

As both acid penetration and chemical release are transport-limited, the model calculates the diffusive transport of stimulant and chemical in the system based on Fickian diffusion

$$
\frac{\partial C}{\partial t}=D_{C} \frac{\partial^{2} C}{\partial x^{2}} \quad \frac{\partial G}{\partial t}=D_{G} \frac{\partial^{2} G}{\partial x^{2}}
$$

(Equations 1, 2)

where $C$ is the concentration of dissolved chemical (with maximum value $C_{\text {sat }}$ at its saturation concentration), $G$ is the concentration of stimulant, $D$ is the diffusion coefficient of the subscript species, $t$ is time and $x$ is distance from the external sink. A complete list of parameters with their corresponding units is given in Table 1. In the barrier membranes, the spatial second derivative is approximated using the centered finite difference method

$$
\left(\frac{\partial^{2} C}{\partial x^{2}}\right)_{i} \cong \frac{C_{i-1}-2 C_{i}+C_{i+1}}{h_{i}^{2}} \quad\left(\frac{\partial^{2} G}{\partial x^{2}}\right)_{i} \cong \frac{G_{i-1}-2 G_{i}+G_{i+1}}{h_{i}^{2}}
$$

(Equations 3, 4)

where $h$ is the thickness of the membrane section represented by node $i$. Equations 3 and 4 are not needed for the node at the interface with the external stimulant source (node 1) as $G_{l}$ is held constant at the external stimulant concentration $G_{u p}$ and $C_{l}$ is held constant at the external chemical concentration 0. No diffusion is possible at the innermost node (node n) until it swells, at which point it delaminates and $G_{n}=G_{u p}, C_{n}=0$.

Approximating $\partial \mathrm{C}_{\mathrm{i}} / \partial \mathrm{t} \approx \Delta \mathrm{C}_{\mathrm{i}} / \Delta \mathrm{t}$ and $\partial \mathrm{G}_{\mathrm{i}} / \partial \mathrm{t} \approx \Delta \mathrm{G}_{\mathrm{i}} / \Delta \mathrm{t}$, the changes in concentration at each node from time $t$ (time step $\mathrm{j}$ ) to time $t+\Delta t$ (time step $\mathrm{j}+1$ ) are calculated from Equations 1-4 as

$$
\Delta C_{i}=D_{C, i} \frac{C_{i-1}-2 C_{i}+C_{i+1}}{h_{i}^{2}} \Delta t \quad \Delta G_{i}=D_{G, i} \frac{G_{i-1}-2 G_{i}+G_{i+1}}{h_{i}^{2}} \Delta t
$$

then the concentrations are updated using the simple explicit Euler method

$$
C_{i, j+1}=C_{i, j}+\Delta C_{i} \quad G_{i, j+1}=G_{i, j}+\Delta G_{i}
$$


For a depot node to swell, its stimulant concentration $G_{i}$ must increase, but this requires a non-zero stimulant diffusion coefficient within the shrunken node. In the physical system, this problem is bypassed by using a weak acid stimulant whose non-dissociated form can partition into the glassy, hydrophobic node and swell it. Rather than track multiple equilibrating forms of the stimulant, however, the computational model calculates the stimulant flux $J_{G}$ and solute flux $J_{C}$ in the depot directly using a forward finite difference approach.

$$
J_{C, i}=D_{C, i} \frac{C_{i-1}-C_{i}}{h_{i}} \quad J_{G, i}=D_{G, i} \frac{G_{i}-G_{i+1}}{h_{i}}
$$

As the chemical is always diffusing in the opposite direction to the stimulant, the nodes for their forward finite difference are reversed. The changes in concentration are then based on the net fluxes into the node.

$$
\Delta C_{i}=\frac{J_{C, i-1}-J_{C, i}}{h_{i}} \Delta t \quad \Delta G_{i}=\frac{J_{G, i-1}-J_{G, i}}{h_{i}} \Delta t
$$

To maintain mass continuity between the barrier layer where the concentration change is calculated directly from the centered finite difference approximation, and the depot layer where concentration change is calculated by the difference in flux (i.e., forward finite difference method) the flux into the last barrier node was subtracted from its concentration change to set the flux from that node into the first depot node.

$$
\begin{aligned}
& J_{C, n b}=J_{C, n b-1}-D_{C, n b} \frac{C_{n b-1}-2 C_{n b}+C_{n b+1}}{h_{n b+1}} \\
& J_{G, n b}=J_{G, n b-1}-D_{G, n b} \frac{G_{n b-1}-2 G_{n b}+G_{n b+1}}{h_{n b+1}}
\end{aligned}
$$

Once diffusive concentration changes are calculated for a given time step, reactive concentration changes are determined. Reactions are considered instantaneous, reducing to two rules. First, stimulant G and scavenger F cannot coexist in the same node. For any node where $F_{i}>0, G_{i}$ will be reduced to 0 and $F_{i}$ will be reduced by the same amount (i.e., 
stoichiometrically). If $G_{i}>F_{i}>0$, then $F_{i}$ is reduced to 0 and $G_{i}$ is reduced stoichiometrically. When $\mathrm{F}=0$ in a depot node, all of the stimuli-sensitive moieties have been stimulated and the depot transitions from the shrunken to the swollen state, with a new set of transport and swelling parameters. Second, all non-shrunken nodes containing solid chemical B are saturated with dissolved chemical. For any node where $B_{i}>0, C_{i}$ will be increased to $C_{\text {sat }}$ and $B_{i}$ will be reduced by the same amount. If $\left(C_{s a t}-C_{i}\right)>B_{i}$, then $B_{i}$ will be reduced to 0 and $C_{i}$ will be increased by that amount.

The chemical concentration at node 1 is held at 0 in equilibrium with the adjacent sink, so the chemical release from the device is simply $J_{C, 2}$, as calculated using Equation 9. This is integrated over time to determine the total chemical released per area, $M_{j}$.

$$
M_{j+1}=M_{j}+J_{C, 2} \Delta t
$$

(Equation 15)

When the system delaminates, the boundary conditions of node 1 are also applied to node $\mathrm{n}$, with the chemical flux into node $\mathrm{n}$ also contributing to $M_{j}$. The timing of this delamination is pegged to the swelling of some percentage of the depot nodes. The annotated delaminating model code is given in the Supplementary Information.

Figure 4 compares the release profiles for models run with different numbers of nodes at parameter values matching an experimental delaminating BMPR system, where delamination occurs when $10 \%$ of the depot nodes are swollen. The ratio of barrier nodes $(n b)$ to depot nodes $\left(n_{d}\right)$ was kept constant at 0.5 . Once $n_{b}=40$, additional nodes do not significantly change the resulting release profile. Computational modeling of delaminating BMPR systems used $n_{b}=60$, $n_{d}=120$. The stability limit for explicit Euler calculations $\left(D_{G} \Delta t / h^{2}<0.5\right)$ demanded $\Delta t<2.7$ $\times 10^{-4} \mathrm{~s}$. Decreasing $\Delta t$ by an order of magnitude had no effect on the profile height to ten 
significant digits. Further modeling used $\Delta t$ values on the order of $10^{-4} \mathrm{~s}$ to allow $D_{G}$ to be varied without losing stability.

\subsubsection{Validation of delaminating BMPR model.}

Several examples of delaminating BMPR release have been reported using the experimental system described in Section 2.2. One of them demonstrates the release of 10 consecutive pulses from a single device(Gandhi et al., 2015). Using the reported physical parameters (listed in the Supplementary Information), the release profile was computationally calculated. Each barrier/depot pair was modeled individually, with the starting time for one pair assigned as the time when the previous pair delaminated. This was set to occur when $10 \%$ of the depot nodes were swollen. The integrated release times for all 10 models were summed to give the overall release profile.

To more subtly test the computational model, release profiles from devices with heterogeneous barriers were calculated. The barriers in these devices contained $40 \% \mathrm{w} / \mathrm{w} \mathrm{ZnO}$ on one side and $0 \% \mathrm{ZnO}$ on the other, oriented with the $\mathrm{ZnO}$ side facing the external sink in one device, and facing the device interior in the other.

In both instances, the time required for the stimulant to penetrate the barrier, lag time $t_{L}$, can be calculated analytically as indicated in Equation 16 and varies linearly with the scavenger concentration, its average depth within the barrier $Z_{m}$ and the square of the barrier's thickness $L$ (Nuxoll et al., 2005).

$$
t_{L}=\frac{L^{2}}{6 D_{G}}\left(1+\frac{6 F Z_{m}}{G_{u p}}\right)
$$

Besides comparing the overall release profiles of the experimental and computational systems, the lag times of each computational model were compared against Equation 16.

\subsubsection{Adaptation of delaminating BMPR model to non-delaminating system.}


By the time the last node of the first depot membrane has swollen, that depot has delaminated and exposed the next barrier membrane to the same boundary conditions experienced by the first barrier. As a result, each barrier/depot pair can be modeled independently and then compiled into a multi-pulse release profile. In non-delaminating systems, however, this is not the case and all the barrier/depot pairs must be modeled together. To construct a five-pulse non-delaminating model, nodes for five barrier membranes and five depot membranes were arrayed together in a single model. Each membrane used the same diffusion and reaction calculations as in the delaminating model, as well as the mass-balance interface between each barrier and its underlying depot. Additionally, a similar mass-balance calculation was used to interface the depot of one barrier/depot pair with the barrier of the next.

In order to study the release of chemical from any particular depot, each depot is loaded with a different solid chemical, meaning that the concentrations of five different chemicals, as well as the stimulant, must be calculated at every node. Moreover, the transport of every chemical must be calculated in every node at every time step for the entire device lifetime. Because of the dramatic increase in computational requirements, the number of nodes in each barrier was decreased from 60 to 20 while maintaining a barrier-to-depot node ratio of 0.5 . Model execution was also moved from individual workstations to a supercomputing cluster. The annotated non-delaminating model code is given in the Supplementary Information, along with an analysis of the effect of node number and time step size. A $\Delta t$ of $5 \times 10^{-3} \mathrm{~s}$ was used for all subsequent modeling, along with a simplified set of parameter values also listed in the Supplementary Information.

\subsection{Investigation of model parameters.}


The parameter space for a non-delaminating BMPR model is tremendous. Even with the assumption that every membrane is initially homogenous, each membrane has diffusion coefficients for the stimulant and each released chemical, initial concentrations of each immobile species, and a thickness. As the number of pulses increases, the possible parameter combinations skyrockets. Moreover, the number of calculations in the non-delaminating BMPR model scales with the square of the number of depots. To narrow the parameter space for evaluation and optimization of BMPR systems, several key parameters were investigated for their effect on pulsatility. In this investigation, each barrier/depot bilayer was initially identical in order to show general trends in pulsatility as a function of the membrane thicknesses, diffusion coefficients, and initial immobile species loadings. These trends should allow more rapid, targeted modeling of non-delaminating BMPR systems.

For the release from a given depot to be pulsatile, it must be completed before the stimulant can trigger release from the next depot. If the time to trigger the next node (plus any barrier nodes in between) is shorter than the time for solute to release, then the release from the nodes will overlap and be continuous. The ratio of these times is therefore an indicator of whether the release from a given node will be pulsatile or continuous. These times, in turn, can be estimated from their corresponding transport parameters.

The time required for chemical in a given node to release from the device ( $\left.t_{\text {solute }}\right)$ is proportional to the amount of solid chemical loaded in the node $(B)$ and its distance to the device interface $(L)$, and inversely proportional to its diffusion coefficient $\left(D_{C}\right)$ and saturation concentration $\left(C_{s a t}\right)$. Similarly, the time required for stimulant to trigger a given node to release $\left(t_{\text {stimulant }}\right)$ is proportional to the amount of scavenger in the node $(F)$ and its distance to the device 
interface $(L)$, and inversely proportional to the stimulant's diffusion coefficient $\left(D_{G}\right)$ and its concentration at the device interface $\left(G_{u p}\right)$.

$$
t_{\text {solute }} \propto \frac{B L}{D_{C} C_{\text {sat }}} \quad t_{\text {stimulant }} \propto \frac{F L}{D_{G} G_{u p}}
$$

(Equations 17, 18)

By taking a ratio of these times, one can estimate the pulsatility of the depot. This ratio is designated as $\Phi$. Combining Equations 17 and 18, $\Phi$ becomes a dimensionless parameter which consolidates six key parameters of the system to indicate the depot pulsatility.

$$
\Phi \equiv \frac{t_{\text {stimulant }}}{t_{\text {solute }}}=\frac{F D_{C} C_{\text {sat }}}{B D_{G} G_{u p}}
$$

Equation 19 suggests that rather than correlating each of these six parameters to the system pulsatility, one need only correlate a single parameter, $\Phi$, regardless of the parameter values forming $\Phi$. This analysis implicitly refers to depot membranes rather than barrier membranes, where B would be zero. In the present analysis, the barriers and depots are assumed to both have the same diffusion coefficients for a given mobile species. As both membranes are highly swollen hydrogels when diffusion is occurring, this assumption is experimentally reasonable; the measured permeabilities of PMMA/DMA and of PVA for methylene blue differ by only $5 \%$ (Gandhi et al., 2015). For the same reasons, the partition coefficients of the mobile species are assumed to be same in each membrane, so $C_{s a t}$ and $G_{u p}$ accurately reflect both membranes. The scavenger concentration in the depot $\left(F_{d}\right)$ is often fixed to provide the desired stimulant sensitivity, but the scavenger concentration in the barrier $\left(F_{b}\right)$ is one of the key design variables which can be easily manipulated. In this analysis, $\Phi$ was calculated using $F_{b}$, and the ratio of the scavenger concentrations $F_{b} / F_{d}$ was fixed at 5.0, similar to the conditions of the experimental non-delaminating BMPR system. Later, $F_{b} / F_{d}$ was systematically varied to determine its effect on system pulsatility. 
The release profile for a non-delaminating BMPR system with five identical pairs of barrier and depot membranes were modeled at $\Phi$ values ranging from 0.3 to 300 by increasing $F_{b}$ (and therefore $F_{d}$ ) while leaving all other parameters constant. Each of the parameters was varied individually at constant $\Phi$ values of 1 and 3 to confirm their interchangability, then the critical $\Phi$ value - the value of $\Phi$ at which the release transitions from pulsatile to continuouswas determined for each depot.

This requires a definition for 'pulsatile' release, arbitrarily defined here as release which is $99 \%$ complete before $1 \%$ of the chemical from the next depot is released. If more than $1 \%$ of the chemical from the next depot is released before $99 \%$ of the chemical from the original depot, then the release has overlapped and is considered continuous.

By increasing the thickness or scavenger concentration of the barriers without altering the depots, the release should become more pulsatile despite a constant $\Phi$. The critical $\Phi$ values $\left(\Phi_{c}\right)$ should therefore change with the ratio of barrier thickness $\left(\ell_{b}\right)$ to depot thickness $\left(\ell_{d}\right)$ and with the ratio of barrier scavenger $\left(F_{b}\right)$ to depot scavenger $\left(F_{d}\right)$. Critical $\Phi$ values were determined at a variety of $\ell_{\mathrm{b}} / \ell_{\mathrm{d}}$ ratios and $F_{b} / F_{d}$ ratios to demonstrate their effect on the critical $\Phi$ values needed for pulsatile release.

\section{Results and Discussion.}

\subsection{Demonstration of physical non-delaminating BMPR system.}

Figure 5 shows schematics and release profiles for a pair of 2-pulse non-delaminating BMPR systems. As anticipated, the profile of the second pulse differs from the first in each trial, with a longer lag time prior to the second pulse and a lower flux during release.

\subsection{Validation of BMPR model.}


For the 10-pulse delaminating BMPR system, the lag times calculated by the model deviated from the analytically predicted lag times by less than $0.0032 \%$, demonstrating the accuracy of model's finite difference calculations. Moreover, the shape of the pulses predicted by the model closely matched those observed experimentally, as shown in Figure 6. Premature delamination of pulses 3-6 due to poor sidewall adhesion shifted the rest of the experimental release profile to the right of the modeled release profile, but in general the model matches not only the lag time, but the shape and duration of the pulsed release, demonstrating that the material assumptions in section 2.3.1 are valid.

This is further confirmed in Figure 7, where the model release profiles for a pair of devices with heterogeneous barriers are compared against experimental results. Again both the timing and the pulse shape of the model prediction are in good agreement with experiment, confirming the validity of the material assumptions.

Besides allowing more refined optimization of BMPR design, this model provides a framework for incorporating more intractable complexities such as finite reaction rates and heterogeneous chemical distribution. Most importantly, it provides a reliable framework for modeling non-delaminating BMPR systems whose behavior cannot be predicted analytically and which require substantially more initial development to obtain even plausible experimental results, such as those shown in Figure 5. Modeling of those non-delaminating profiles is discussed in the Supplementary Information.

\subsection{Non-delaminating BMPR modeling.}

\subsubsection{Correlation with $\Phi$.}

Section 2.4 postulated that the effects of several critical design parameters could be consolidated into a single dimensionless group, $\Phi$, the ratio of characteristic transport times for 
the stimulant and the solute. As $\Phi$ decreases, the release profile would transition from pulsatile to continuous, regardless of which parameter changed to decrease $\Phi$. Figure 8 demonstrates this with $\Phi$ varying from 0.3 to 300 by increasing the scavenger loading $F$. This increased the time scale for release, as shown on the left-hand side of the figure, but also changed the shape of the release profile, as shown on the right-hand side where the time axis of each profile is normalized by the time required for that profile to reach complete (99.9\%) release. There is a clear transition from continuous release at low $\Phi$ values to discrete pulses at large $\Phi$ values.

All of the six key parameters comprising $\Phi$ have the same effect on pulsatility, as shown in Figure 9, where the $\Phi=1$ profile from Figure 8 is plotted against other $\Phi=1$ profiles obtained by changing other parameters with a commensurate change in $F$. The right-hand side of Figure 9 demonstrates that not only is the release continuous, its behavior is comparable to release from a uniform monolith with a square-root dependence on time. At $\Phi=1$, the barriers have no significant effect on pulsatility. At $\Phi=3$, on the other hand, each depot releases a discrete pulse of chemical, regardless of the parameter combination forming $\Phi$ (Figure 10).

There are a few key assumptions made in this analysis, notably that the amount of stimulant needed to simply create the concentration gradient in the device is negligible compared to the amount consumed by the scavenger, and that virtually all of the solute flux is driven by a concentration difference of $C_{s a t}$ at the source node to 0 at the device interface. The degree to which $F>>G_{u p}$ and $B>>C_{s a t}$ is discussed in the Supplementary Information.

Figures 8-10 show qualitatively the relationship between $\Phi$ and pulsatility, but for a quantitative correlation, pulsatile release must be precisely defined. For this investigation, the release from a given depot is deemed to be pulsatile if it is $99 \%$ complete before $1 \%$ of the chemical from the next depot is released. By iterative modeling, the value of $\Phi$ which yields that 
precise amount of overlap was determined. This critical $\Phi$ value, $\Phi_{c}$, increases with the number of depots, as shown in Figure 11. As later pulses diffuse through more spent layers in the device, they broaden, requiring them to initially be more separated in order to escape the device separately. Plotting $\Phi_{\mathrm{c}}$ vs. the number of pulses on a log-log scale shows that $\Phi_{\mathrm{c}}$ increases with pulse number to the 0.58 power with an $\mathrm{r}^{2}$ of 0.99996 when the barrier and depot membrane thicknesses are equal $\left(\ell_{\mathrm{b}} / \ell_{\mathrm{d}}=1.0\right)$. By extrapolation, one can quickly estimate whether pulsatile release is feasible for a given set of materials in a much larger stack of depots.

\subsubsection{Correlation with membrane thickness.}

Membrane thickness plays a critical role in BMPR pulse timing. As seen in Equation 16, the time required for stimulant to penetrate a membrane varies with the square of the membrane's thickness. Thickness cancels out of the expression for $\Phi$ in Equation 19, however, and computational modeling of a 5-pulse non-delaminating BMPR device demonstrates that thickness has no effect on $\Phi_{c}$, providing yet another means of manipulating the release profile timing independently from the release profile shape. The ratio of barrier thickness $\ell_{b}$ to depot thickness $\ell_{\mathrm{d}}$, on the other hand, should have a strong effect, where doubling the barrier thickness increases the lag time between pulses by a factor of four. One might expect, then, that $\Phi_{c}$ would vary inversely with the square root of $\ell_{\mathrm{b}} / \ell_{\mathrm{d}}$, as confirmed in Figure 12. Importantly, Figure 11 also shows that the extrapolation to larger pulse numbers also changes slightly as the order of magnitude of $\ell_{\mathrm{b}} / \ell_{\mathrm{d}}$ changes.

\subsubsection{Correlation with scavenger loading.}

The role played by the ratio of scavenger concentrations in the barrier vs. the depot is more complicated, however. In most systems, the stimuli-sensitive moieties in the depot membrane will effectively consume stimulant in order to trigger release from the depot, so both 
the depots and the barriers have effective scavenger concentrations, $F_{d}$ and $F_{b}$, respectively. In these studies, $\Phi$ has been calculated using the barrier scavenger concentration $F_{b}$. If $F_{d}$ is reduced without changing $F_{b}$, then the calculated $\Phi$ is unchanged but stimulant penetrates the depot more quickly. The consequent effect on system pulsatility depends on which process is rate limiting. If solute release is limited by how quickly the depot is stimulated, then faster penetration of the depot will result in a shorter pulse duration but it will not change the time between the end of one pulse and the beginning of the next; $\Phi_{c}$ will not change. On the other hand, if solute release is limited by dissolution and diffusion of the solute, then the pulse duration will still be just as long, but the next pulse will start sooner since the stimulant begins penetrating the next barrier sooner. The $\Phi$ required for the release to be pulsatile $\left(\Phi_{c}\right)$ must increase.

This relationship between $\Phi_{\mathrm{c}}$ and $F_{\mathrm{b}} / F_{d}$ holds true regardless of which scavenger concentration is changed. Experimentally, $F_{b}$ is typically much easier to vary over a wide concentration range than $F_{d}$. In Figure $13, F_{d}$ is held constant at $0.157 \mathrm{M}$ while $\mathrm{F}_{\mathrm{b}}$ is changed to vary the $F_{b} / F_{d}$ ratio and $G_{u p}$ is varied to determine the consequent $\Phi_{\mathrm{c}}$. When $F_{b}<<F_{d}\left(\log F_{b} / F_{d}\right.$ negative), its effect on $\Phi_{\mathrm{c}}$ is large, while for positive $\log F_{b} / F_{d}$ values, further increases in $F_{b} / F_{d}$ have less effect on $\Phi_{\mathrm{c}}$. Figure 13 also demonstrates that the effect of the scavenger ratio is coupled to the thickness ratio. When the depot is much thinner than the barrier, it plays a smaller role in the time required for the stimulant to trigger the next pulse, and therefore a smaller role in increasing $\Phi_{\mathrm{c}}$. For $\ell_{\mathrm{b}} / \ell_{\mathrm{d}}=5$, Figure 13 shows that once $F_{b}>F_{d}$, further increases to $F_{b}$ have little effect on $\Phi_{c}$, and instead only alter the absolute pulse timing. When $\ell_{b} / \ell_{d}=0.2$, on the other hand, the $F_{b} / F_{d}$ ratio has a large effect on pulsatility even at high values. Due to this interdependence, simultaneous exploration of both $\ell_{\mathrm{b}} / \ell_{\mathrm{d}}$ and $F_{b} / F_{d}$ is not analytically feasible, particularly for extrapolation to a large number of pulses. 


\subsubsection{Implications.}

Barrier-Mediated Pulsatile Release offers many capabilities not available through any other passive delivery system. Despite the rudimentary demonstration in Section 3.1, however, development of experimental non-delaminating BMPR systems remains challenging, with a significant initial investment required to determine the appropriate fabrication procedures for a given application. Such investment is unlikely without a separate, accessible means of first evaluating the feasibility of achieving the target release profile with the proposed materials. While many of the most important performance parameters can be predicted analytically for a delaminating BMPR system, the non-delaminating system is too complex for tractable analytical modeling. This computational model, then, is critical not only for the design and development of any non-delaminating BMPR system, but for initial feasibility assessments.

If the application requires many pulses, even computational modeling becomes a significant investment, given the enormous parameter space involved. Correlations which can quickly indicate whether the release from a particular depot will form a discrete pulse or overlap with release from other depots are therefore valuable assessment tools, along with trends indicating the effects of changing various design parameters, most notably the scavenger loading and thickness of the barrier membranes.

\section{Conclusions.}

Pulsatile chemical delivery is important in a variety of fields, but automation of pulsatile delivery is typically complex and expensive compared to sustained release, which may be achieved via formulation. Barrier-Mediated Pulsatile Release offers an elegant, materials-based approach to pulsatile delivery, transducing a constant stimulant signal into a pulsatile solute signal using only a sealed stack of polymer films. Delaminating BMPR systems may deliver an 
arbitrarily large number of discrete pulses, each with its own chemical identity and pulse timing. Barrier-Mediated Pulsatile Release can also be achieved without delamination, as demonstrated experimentally for the first time here. In a non-delaminating system the number of discrete pulses at a given periodicity is inherently limited, however, and prediction of this limit is analytically intractable. A computational model has been constructed and validated against delaminating BMPR devices, then adapted for non-delaminating BMPR systems. Using this model, the ability to deliver a given number of discrete pulses has been correlated to several key design parameters including the diffusion coefficients, chemical loading, and upstream stimulant concentration of the system, as well as the thicknesses and scavenger loadings of the layers. This model, guided by these correlations, should allow quick evaluation of the feasibility of any nondelaminating BMPR application, as well as guide later optimization and development.

\section{Acknowledgements.}

The authors gratefully acknowledge the University of Iowa, the National Science Foundation (CBET-1133297), and the American Heart Association (11SDG7600044) for their support of this work.

\section{References.}

Abrams, J., 1988. A reappraisal of nitrate therapy. Journal of the American Medical Association $259,396-401$.

Bhalla, A.S., Siegel, R.A., 2014. Mechanistic studies of an autonomously pulsing hydrogel/enzyme system for rhythmic hormone delivery. Journal of Controlled Release 196, 261-271.

Brabant, G., Prank, K., Schöfl, C., 1992. Pulsatile patterns in hormone secretion. Trends in Endocrinology and Metabolism 3, 183-190.

Centers for Disease Control and Prevention, 2009. Rabies Vaccine: What You Need to Know. www.cdc.gov/vaccines/pubs/vis/downloads/vis-rabies.pdf.

Firestone, B.A., Siegel, R.A., 1988. Dynamic pH-dependent swelling properties of a hydrophobic polyelectrolyte gel. Polymer Communications 29, 204-208. 
Firestone, B.A., Siegel, R.A., 1991. Kinetics and mechanisms of water sorption in hydrophobic, ionizable copolymer gels. Journal of Applied Polymer Science 43, 901-914.

Fujioka, K., Sato, S., Takada, Y., Sasaki, Y., Tamura, N., 1987. Sustained pulsewise release pharmaceutical preparation, European Patent no. 230654 p. 12.

Gandhi, S., Gosse, M.D., Nishii, Y., Nuxoll, E., 2015. Barrier-mediated pulsatile release. Journal of Membrane Science. Journal of Membrane Science 495, 351-360.

Gazzaniga, A., Palugan, L., Foppoli, A., Sangalli, M.E., 2008. Oral pulsatile delivery systems based on swellable hydrophilic polymers. European Journal of Pharmaceutics and Biopharmaceutics 68, 11-18.

Hayes, F.J., Crowley, W.F.J., 1998. Gonadotropin Pulsations across Development. Hormone Research 49, 163-168.

Innominato, P.F., Roche, V.P., Palesh, O.G., Ulusakarya, A., Spiegel, D., Levi, F.A., 2014. The circadian timing system in clinical oncology. Annals of Medicine 46, 191-207.

Jeon, J.H., Puleo, D.A., 2008. Alternating release of different bioactive molecules from a complexation polymer system. Biomaterials 29, 3591-3598.

Jiang, H.L., Zhu, K.J., 2000. Pulsatile protein release from a laminated device comprising of polyanhydrides and $\mathrm{pH}$-sensitive complexes. International Journal of Pharmaceutics 194, 51-60.

Lévi, F., Zidani, R., Misset, J.-L., 1997. Randomised multicentre trial of chronotherapy with oxaliplatin, fluorouracil, and folinic acid in metastatic colorectal cancer. The Lancet 350, 681-686.

Li, J.T., Lockey, R.F., Bernstein, I.L., Portnoy, J.M., Nicklas, R.A., 2003. Allergen Immunotherapy: a practice parameter. Annals of Allergy, Asthma \& Immunology 90, 140.

Liu, X., Pettway, G.J., McCauley, L.K., Ma, P.X., 2007. Pulsatile release of parathyroid hormone from an implantable delivery system. Biomaterials 28, 4124-4131.

McKim, W.A., 2007. Drugs and Behavior. An Introduction to Behavioral Pharmacology.

Nuxoll, E.E., Cussler, E.L., 2005. The third parameter in reactive barrier films. AICHE Journal $51,456-463$.

Nuxoll, E.E., Siegel, R.A., Cussler, E.L., 2005. Layered reactive barrier films. Journal of Membrane Science 252, 29-36.

Siegel, R.A., Firestone, B.A., 1988. pH-dependent equilibrium swelling properties of hydrophobic polyelectrolyte copolymer gels. Macromolecules 21, 3254-3259.

Tang, E.S.K., Chan, L.W., Heng, P.W.S., 2005. Coating of multiparticulates for sustained release. American Journal of Drug Delivery 3, 17-28. 
Thitinan, S., McConville, J.T., 2012. Development of a gastroretentive pulsatile drug delivery platform. Journal of Pharmacy and Pharmacology 64, 505-516.

Walters, A.A., Krastev, C., Hill, A.V., Milicic, A., 2015. Next generation vaccines: single-dose encapsulated vaccines for improved global immunisation coverage and efficacy. Journal of Pharmacy and Pharmacology 67, 400-408. 


\section{Figure Captions:}

Figure 1. Delaminating vs. Non-Delaminating BMPR Systems. In the delaminating system, every barrier layer is sequentially exposed to the same stimulant/solute sink. In the nondelaminating system, every barrier and depot experiences a different flux of stimulant and solute due to the presence of spent barriers and depots.

Figure 2. Non-Delaminating BMPR Systems. At right, a side-view schematic of the device design. At right, an overhead view of an actual device. Note that the schematic is not drawn to scale; the aspect ratio of the device is similar to a coin.

Figure 3. Schematic of Single-Pulse BMPR System for Computational Model. The barrier membrane is divided into $\mathrm{n}_{\mathrm{b}}$ segments, each represented by a node on it upstream edge (i.e., the edge nearest the external sink. Similarly, the depot is divided into $\mathrm{n}_{\mathrm{d}}$ segments and nodes. An additional node is located at the downstream edge of the device to provide a boundary condition. Additional membrane pairs may be inserted before this innermost node.

Figure 4. Node Sensitivity of Computational Model. Error is proportional to inverse of node number; at $\mathrm{n}_{b}=40, \mathrm{n}_{d}=80$, maximum error is $9.2 \times 10^{-10} \%$ of total solute released.

Figure 5. Non-delaminating Barrier-Mediated Pulsatile Release. Left: Schematic of the first non-delaminating BMPR system. Stimulant enters through wire mesh on top, eventually triggering swelling of depot layers, which push down on poly(tetrafluoroethylene) (PTFE) plug, deforming rubber cement bottom, but remaining intact. Right: Release profile of device (solid line) and replicate (dashed). Note that schematic is not drawn to scale; the aspect ratio of the device is similar to a coin.

Figure 6. Validation of Computational Model on 10-pulse Delaminating BMPR System. Model correctly calculates not only lag time (available analytically) but also pulse shape. Deviation in pulses 3-6 is due to premature delamination in the experimental system reported in [Gandhi, 2015].

Figure 7. Validation of Computational Model on Delaminating BMPR Systems with Heterogeneous Barriers. Model correctly calculates lag time for barriers with non-homogenous initial distributions of scavenger. Experimental data taken from [Gandhi, 2015].

Figure 8. Effect of $\Phi$ on Release Profile. As scavenger concentration F increases, thereby increasing $\Phi$, pulses take longer to release, as shown on the left-hand-side, and become more distinct, as shown in the time-normalized chart on the right-hand-side. Times in that chart are normalized by the time required for $99.9 \%$ of the solute to release from that sample. The same legend applies to both charts.

Figure 9. Universality of $\Phi$ for continuous release. The shape of the release profile is uniform at $\Phi=1$ regardless of the parameter combination used to achieve that $\Phi$. In these profiles, $\Phi$ is held equal to 1 by adjusting $\mathrm{F}$ to compensate for the factor change indicated in the legend. For instance, for "DG $\uparrow 10 x$ " the stimulant diffusion coefficient is increased by a factor of 10 from the Control, and $\mathrm{F}$ is also increased by a factor of 10. At right, the total release is linear with the square root of time, consistent with transport-limited release. 
Figure 10. Universality of $\Phi$ for pulsatile release. The shape of the release profile is uniform at $\Phi=3$ regardless of the parameter combination used to achieve that $\Phi$. In these profiles, $\Phi$ is held equal to 3 by adjusting $F$ to compensate for the factor change indicated in the legend. For instance, for "DG $\uparrow 10 \mathrm{x}$ " the stimulant diffusion coefficient is increased by a factor of 10 from the Control, and $\mathrm{F}$ is also increased by a factor of 10. At right, the timing of each pulse is uniform when plotted against the square root of time, consistent with transport-limited release.

Figure 11. Determination of $\boldsymbol{\Phi}_{\mathbf{c}}$ for large numbers of pulses. The limiting $\Phi_{c}$ value, below which over $1 \%$ of the solute release from adjacent pulses overlap, increases with the number of pulses, closely tracking a power-law relationship. The exponent of the relationship varies slightly with the ratio of the barrier and depot thicknesses, $\ell_{\mathrm{b}} / \ell_{\mathrm{d}}$. Note that the Pulse Number here is the number of the earlier pulse of any adjacent pair.

Figure 12. Effect of barrier:depot thickness ratio. While the absolute thickness of the membranes has no effect on the critical $\Phi$ value, $\Phi_{c}$ does have a square-root dependence of the ratio of the membrane thicknesses.

Figure 13. Effect of barrier:depot scavenger concentration ratio. At low $\mathrm{F}_{b} / \mathrm{F}_{\mathrm{d}}$ values, increasing the scavenger concentration in the barrier relative to the depot actually decreases the pulsatility of the system (increases the $\Phi_{c}$ required for pulsatile release). At high $\mathrm{Fb}_{b} / \mathrm{F}_{\mathrm{d}}$ values, this effect diminishes, at a rate dependent on the thickness ratio $\ell_{\mathrm{b}} / \ell_{\mathrm{d}}$. 

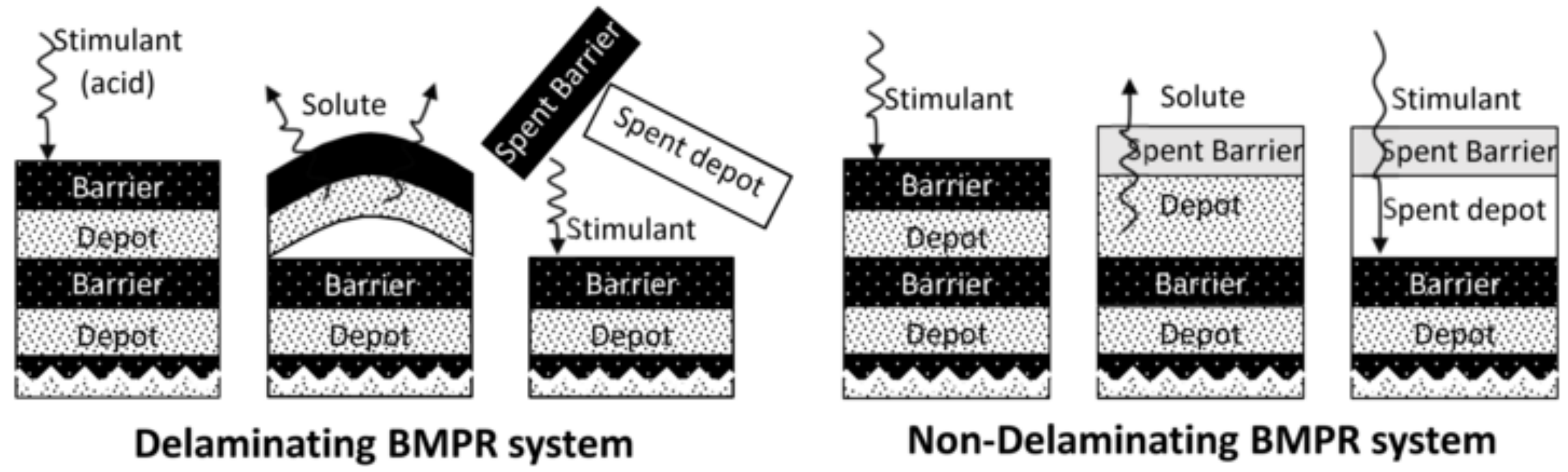

Non-Delaminating BMPR system 
Metal mesh filter
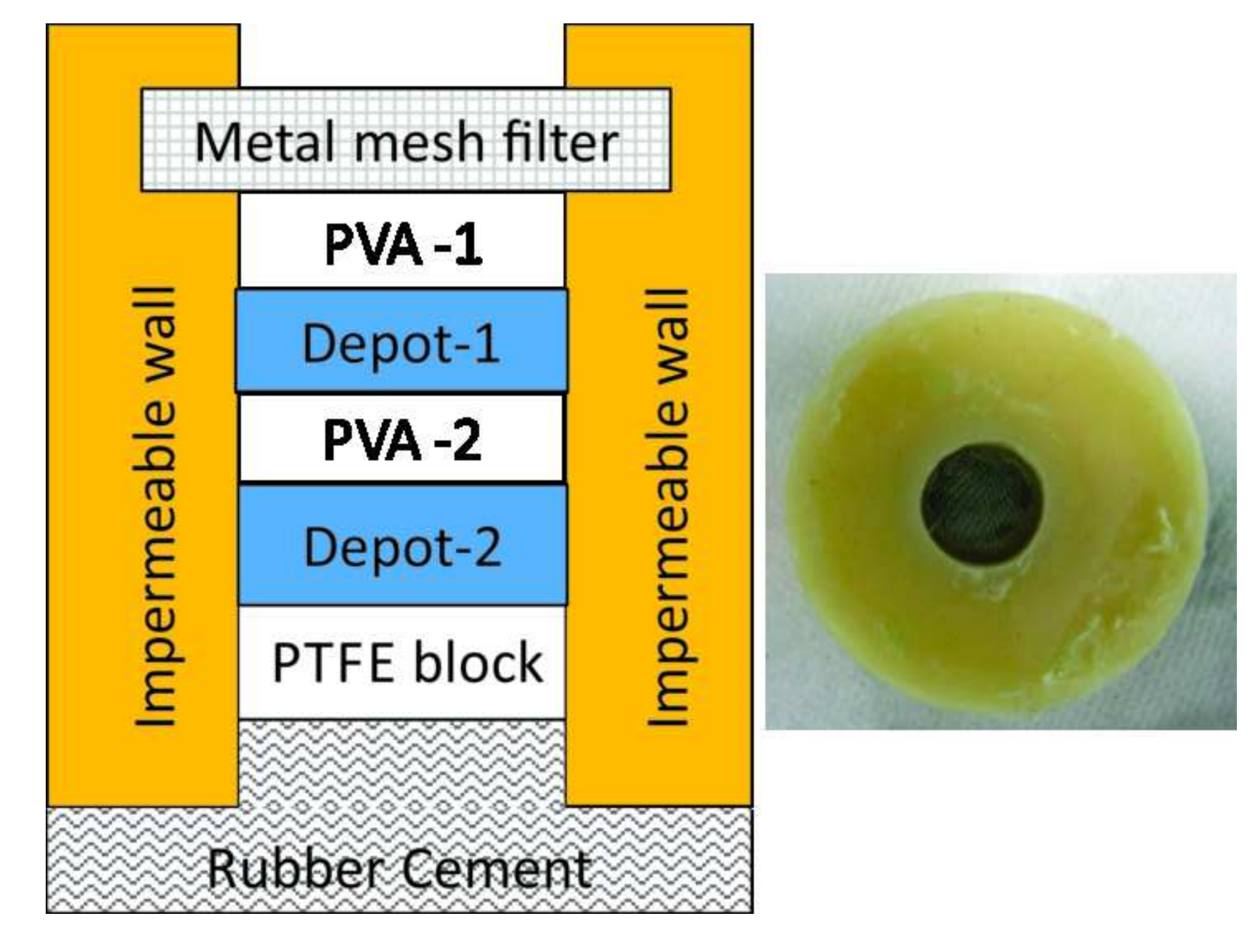
$\mathbf{p}^{\prime}$
$\mathrm{d}$

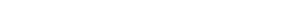




\section{External Sink}

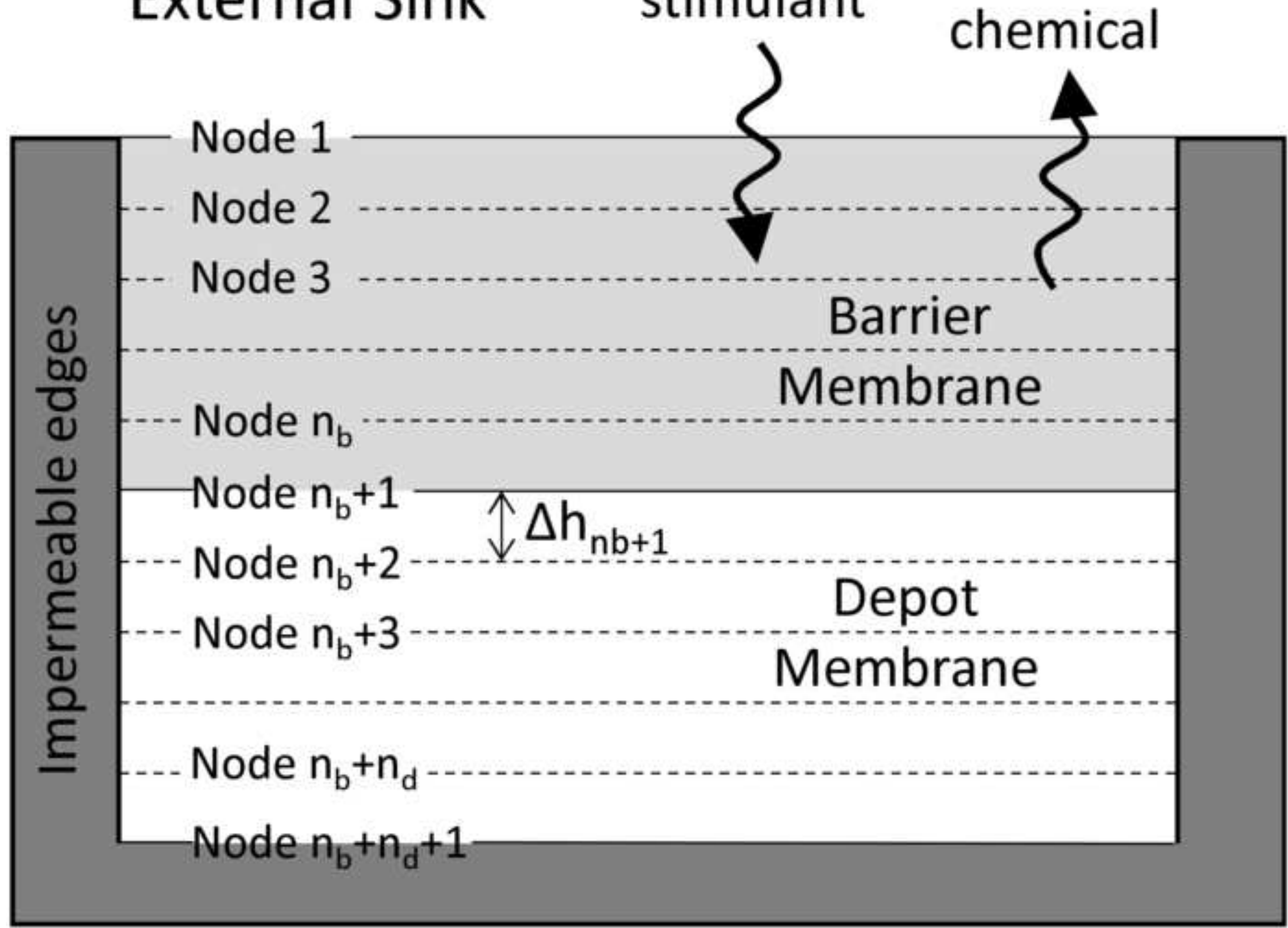




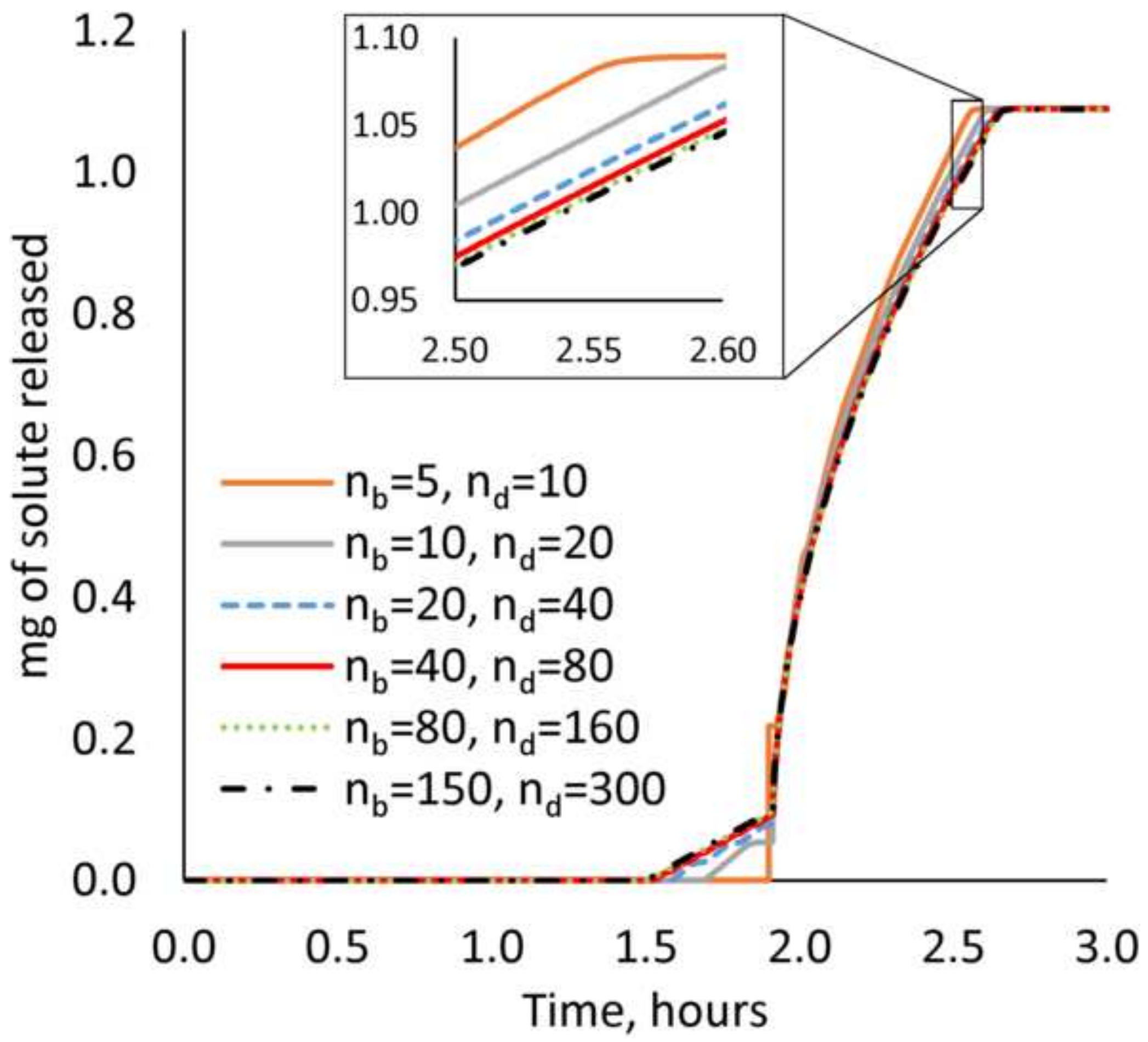



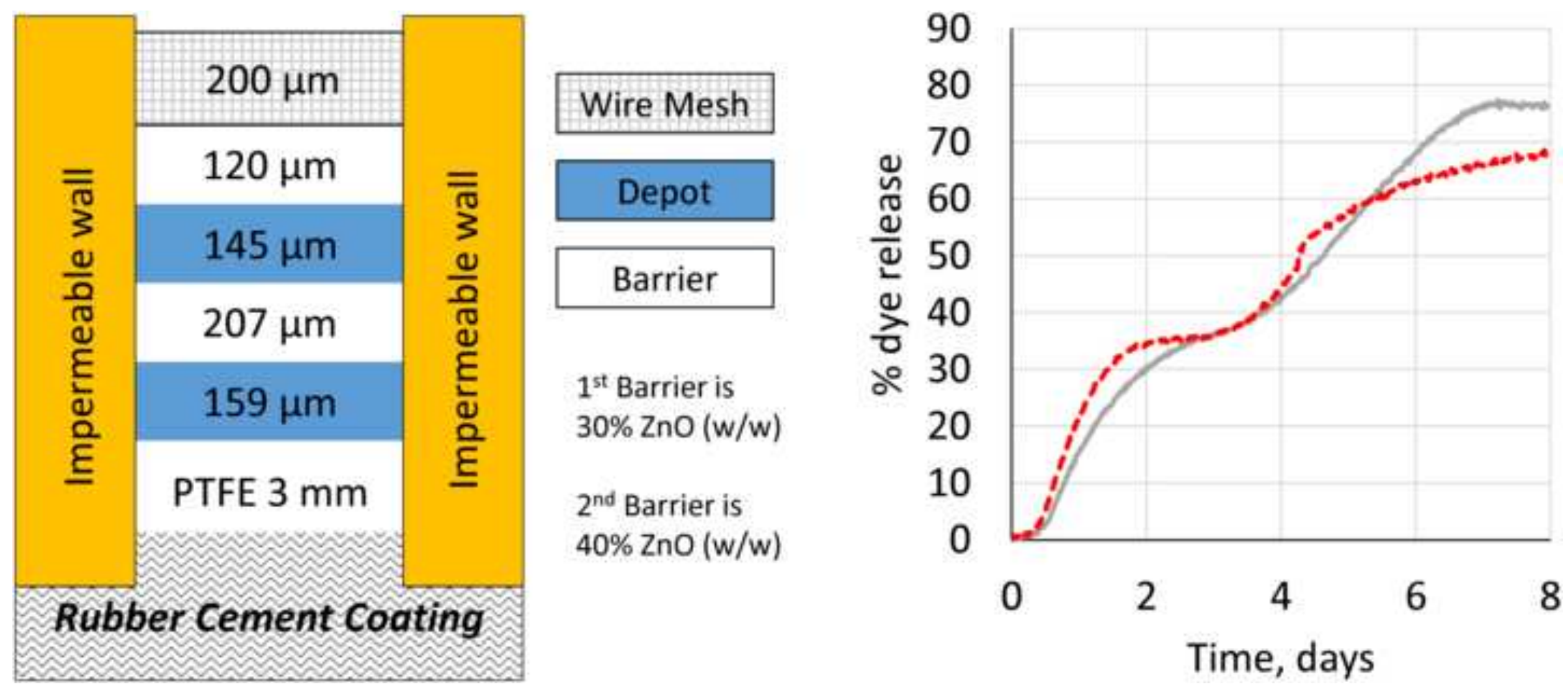


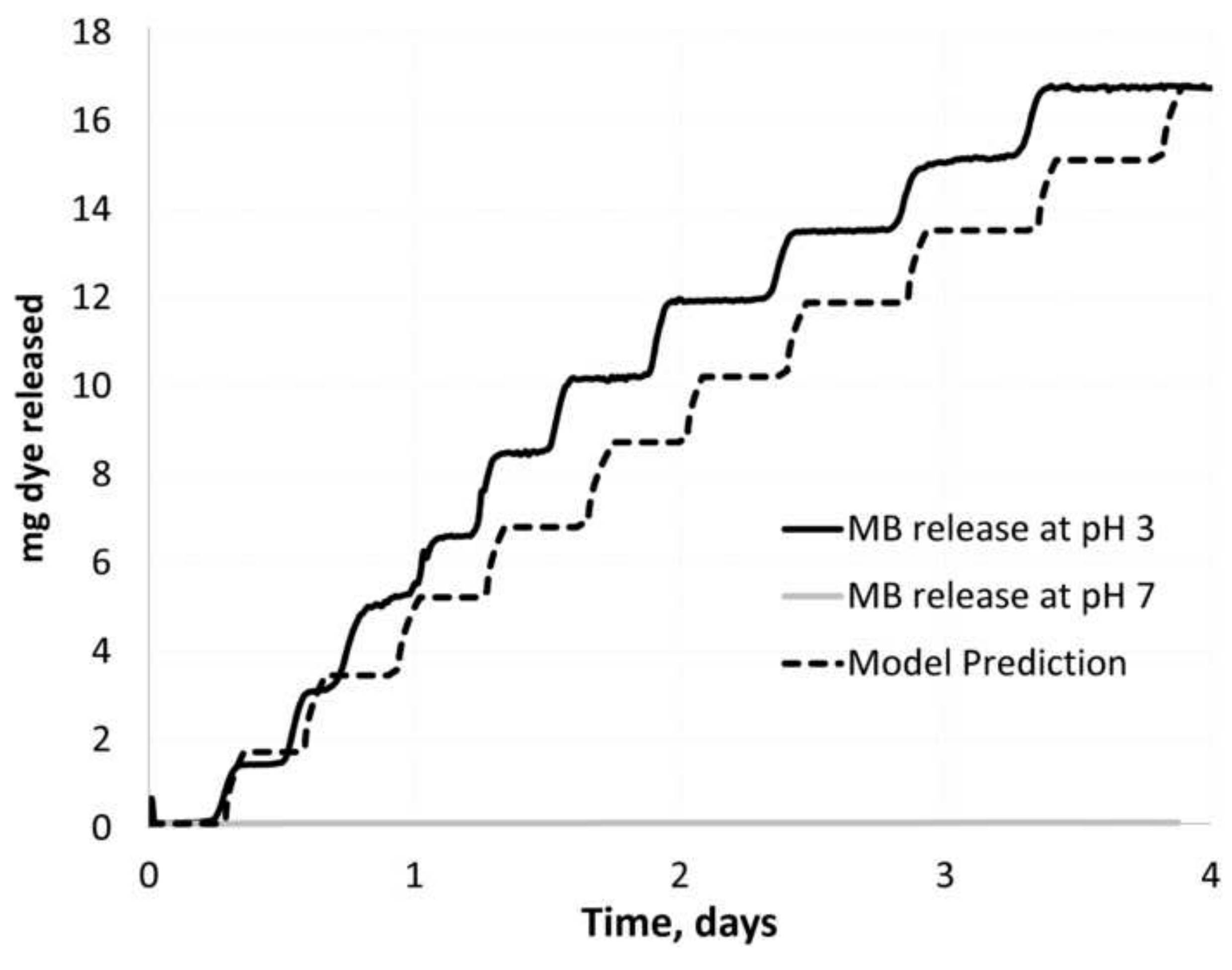



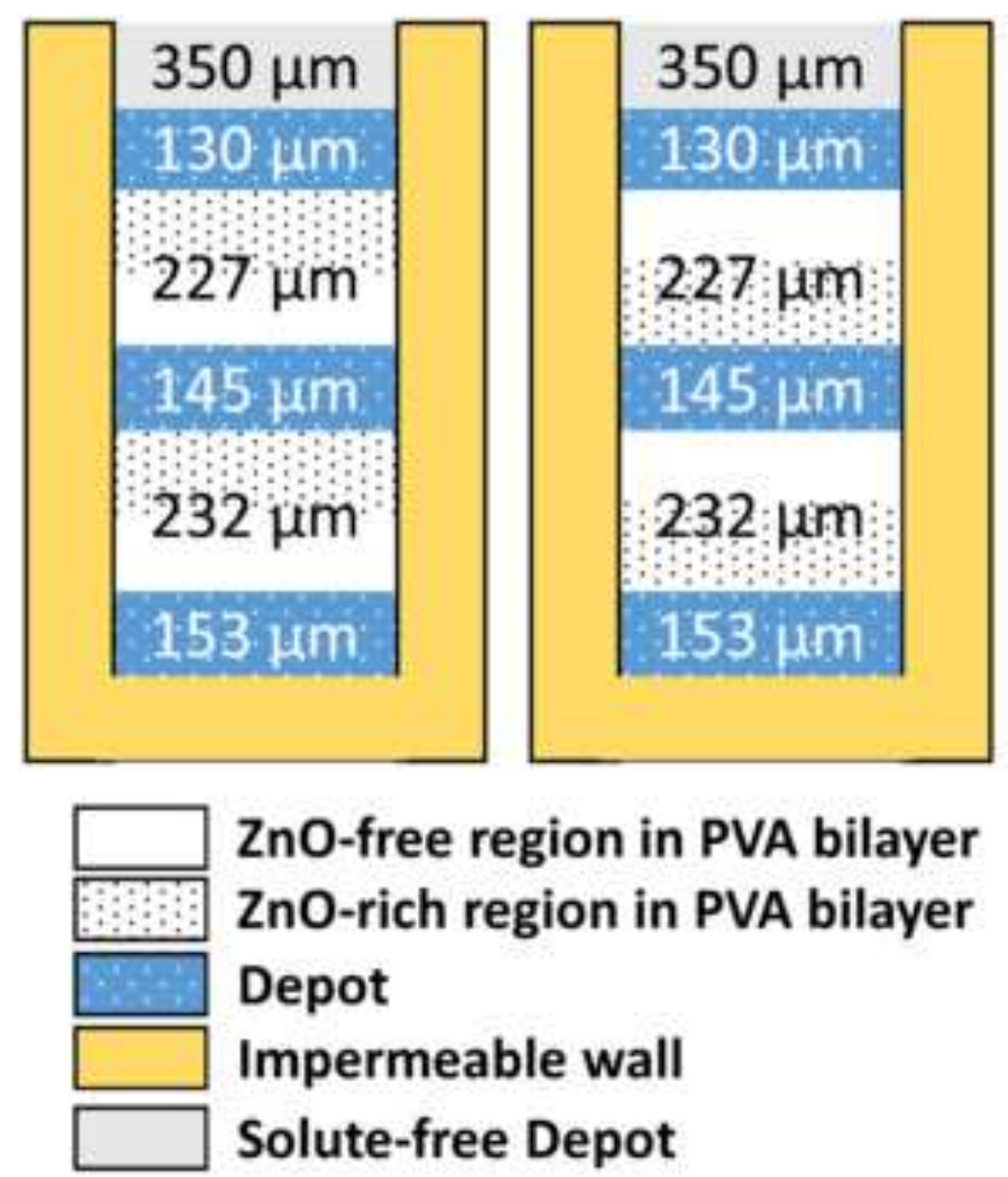

ZnO-free region in PVA bilayer ZnO-rich region in PVA bilayer Depot Impermeable wall Solute-free Depot

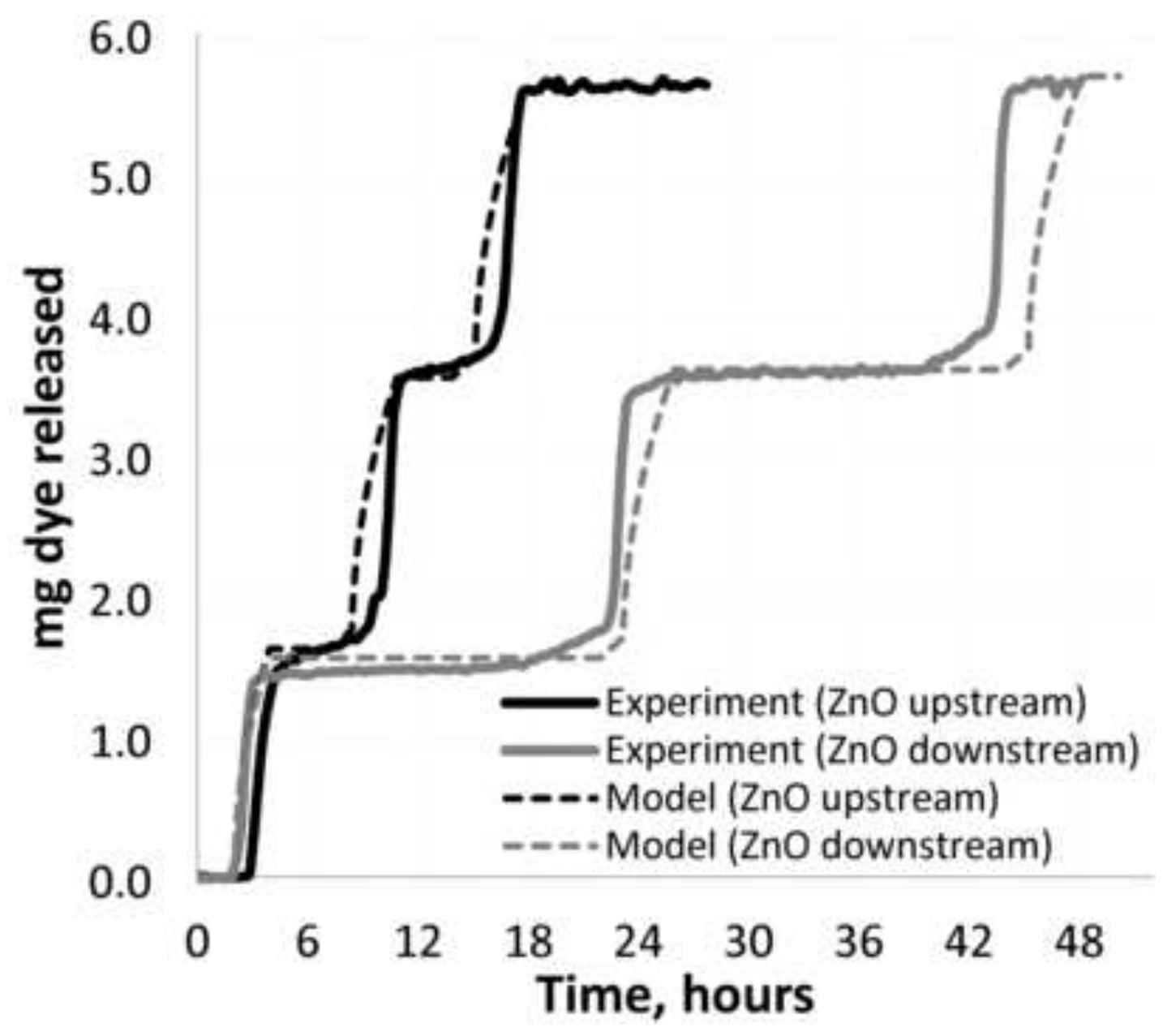



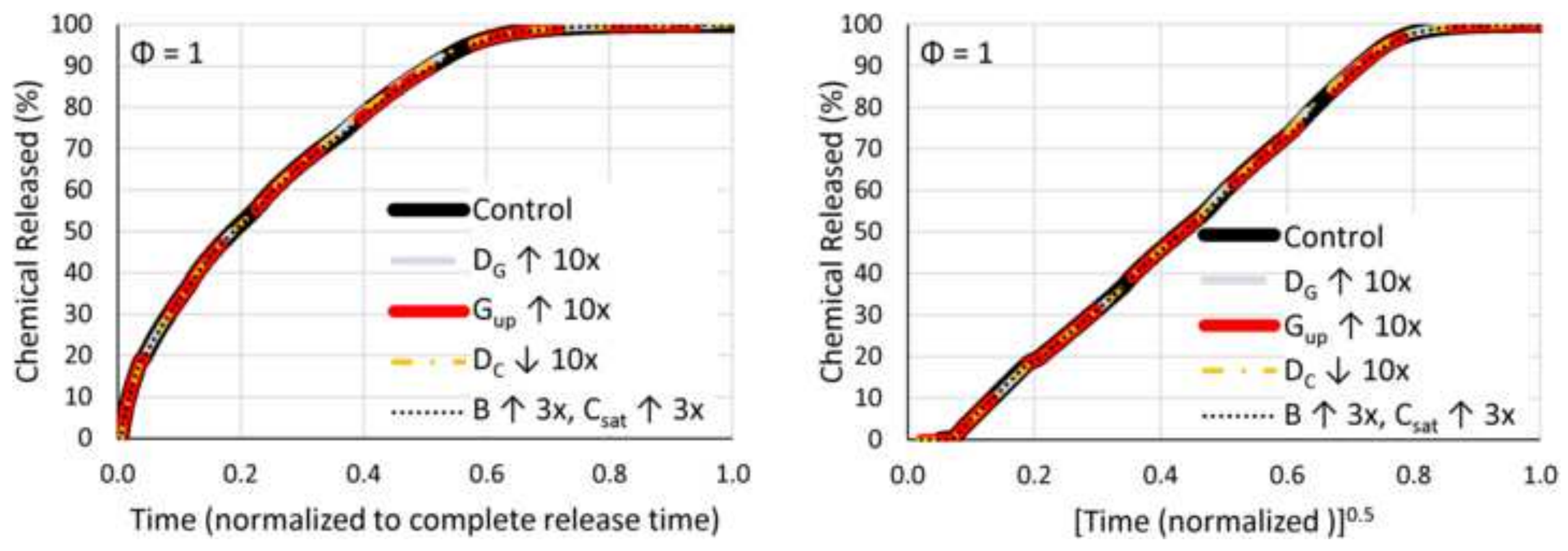

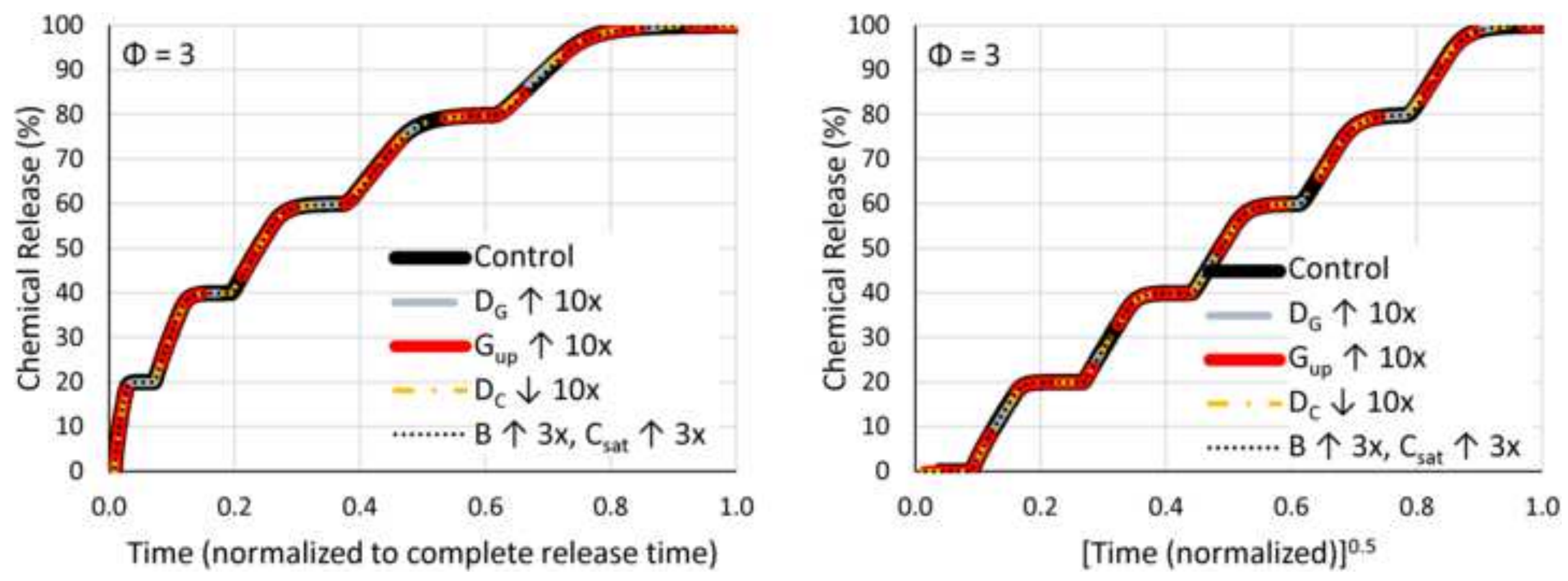


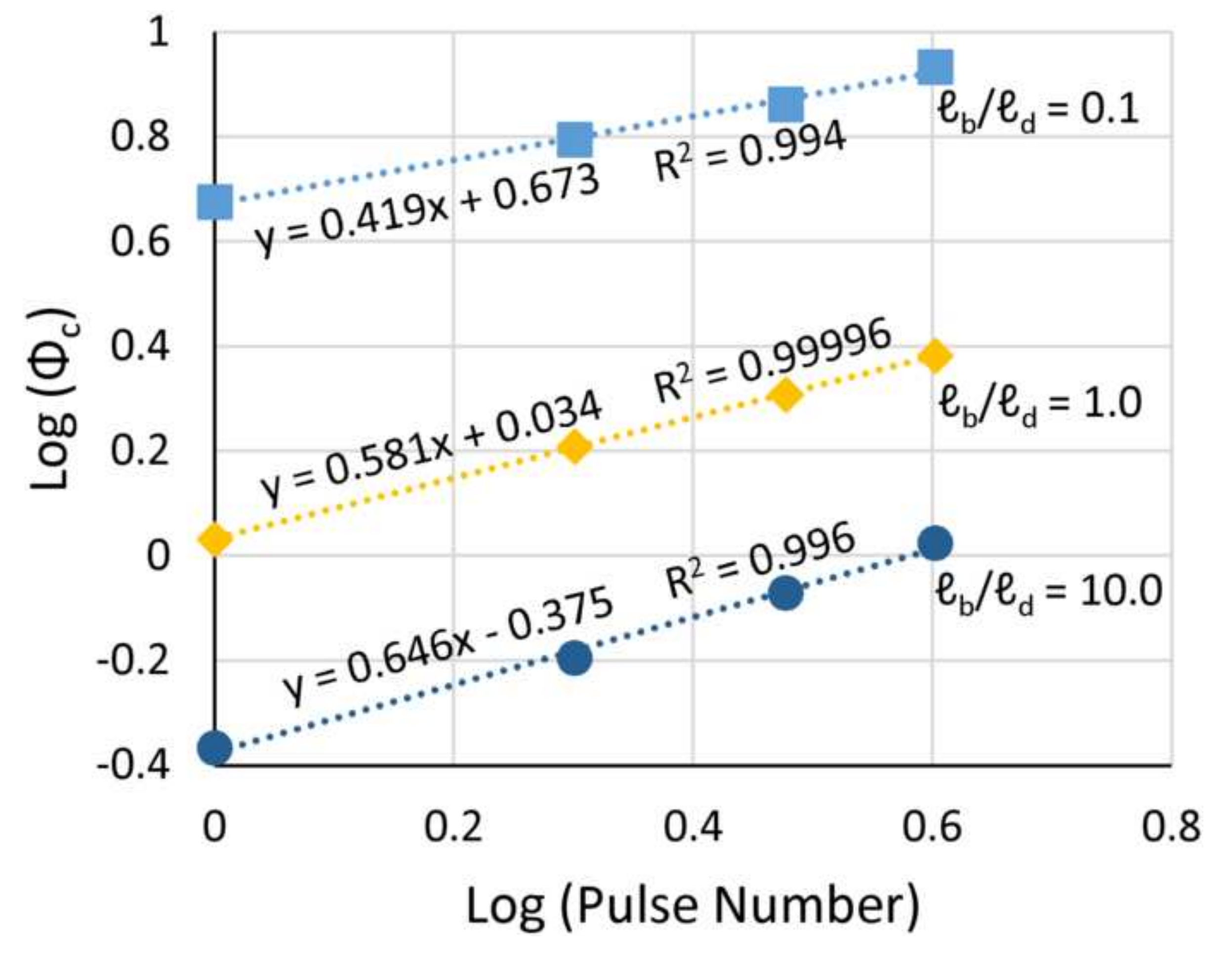

.

. 

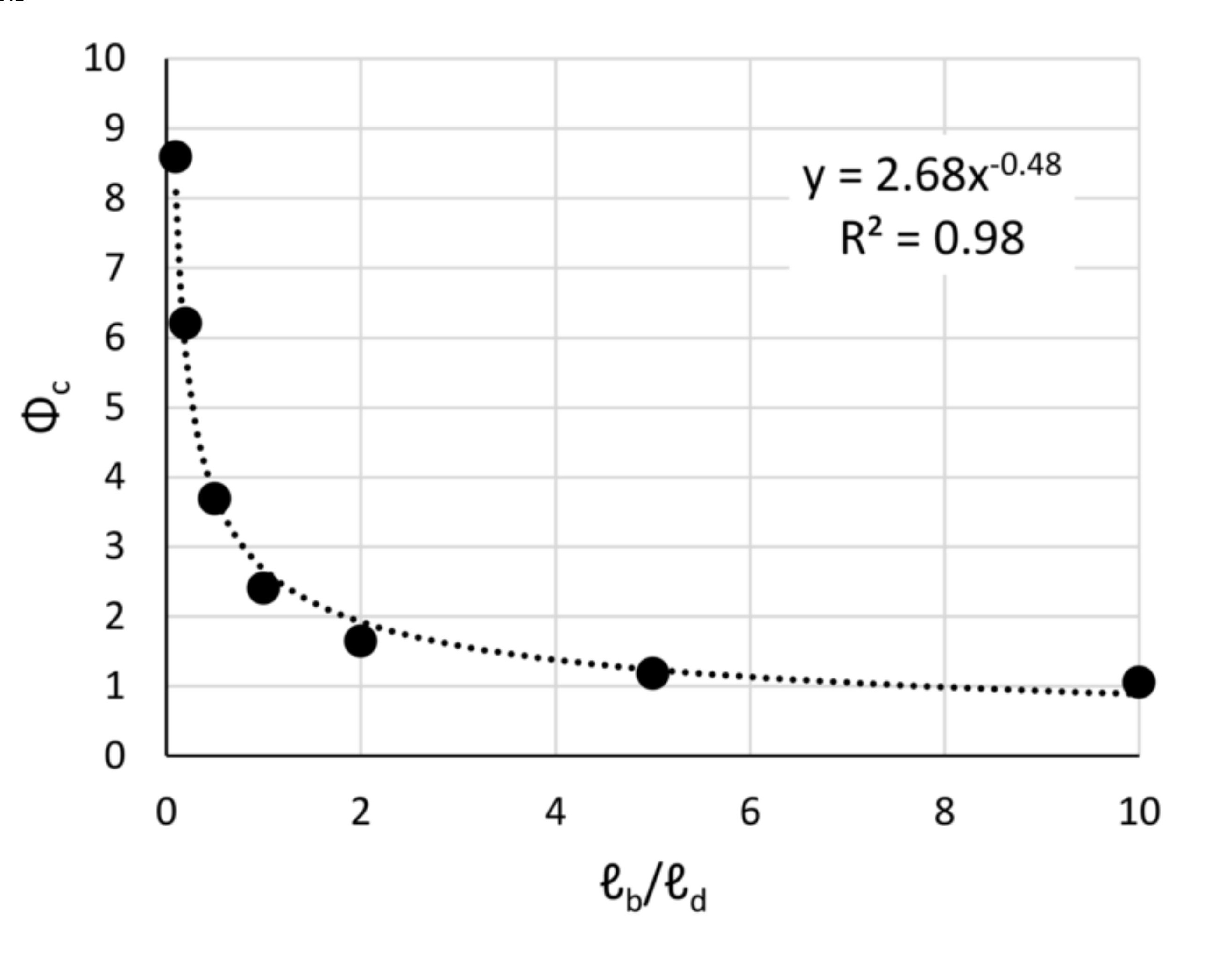


\begin{tabular}{|c|c|c|}
\hline Symbol & Description & Dimensions \\
\hline$B$ & Concentration of solid payload chemical & $\mathrm{M} \mathrm{L}^{-3}$ \\
\hline C & Concentration of dissolved payload chemical & $\mathrm{M} \mathrm{L}^{-3}$ \\
\hline$C_{\text {sat }}$ & Saturation concentration of dissolved payload chemical & $\mathrm{M} \mathrm{L}^{-3}$ \\
\hline$D_{C}$ & Diffusion coefficient of dissolved payload chemical & $\mathrm{L}^{2} \mathrm{t}^{-1}$ \\
\hline$D_{G}$ & Diffusion coefficient of stimulant & $\mathrm{L}^{2} \mathrm{t}^{-1}$ \\
\hline$F$ & Concentration of scavenger & $\mathrm{M} \mathrm{L}^{-3}$ \\
\hline$G$ & Concentration of stimulant & $\mathrm{ML}^{-3}$ \\
\hline$G_{\text {up }}$ & Concentration of stimulant at external device interface & $\mathrm{ML}^{-3}$ \\
\hline$h$ & Node thickness & $\mathrm{L}$ \\
\hline$J$ & Molar flux & $M L^{-2} t^{-1}$ \\
\hline$L$ & Distance to external device interface & $\mathrm{L}$ \\
\hline e & Membrane thickness & $\mathrm{L}$ \\
\hline M & Cumulative mass of released payload chemical per area & $\mathrm{M} \mathrm{L}^{-2}$ \\
\hline$n_{b}$ & Number of barrier nodes & - \\
\hline$n_{d}$ & Number of depot nodes & - \\
\hline$t$ & Time & $\mathrm{t}$ \\
\hline$t_{L}$ & Lag time & $\mathrm{t}$ \\
\hline$x$ & Spatial coordinate & $\mathrm{L}$ \\
\hline$Z_{m}$ & Scavenger center of mass & - \\
\hline$\Phi$ & Pulsatility factor & - \\
\hline \multirow[t]{2}{*}{$\Phi_{c}$} & Critical pulsatility value & - \\
\hline & & $\mathrm{M}[=]$ mass (moles) \\
\hline \multicolumn{2}{|c|}{$\underline{\text { Subscripts }}$} & $\mathrm{L}[=]$ length $(\mathrm{cm})$ \\
\hline$b$ & Barrier & $\mathrm{t}[=]$ time $(\mathrm{s})$ \\
\hline C & Dissolved payload chemical & \\
\hline$d$ & Depot & \\
\hline G & Stimulant & \\
\hline$i$ & Node index & \\
\hline r & Timestep index & \\
\hline
\end{tabular}

